\title{
Editorial: Beauty 2019 at Ljubljana, Slovenia
}

\section{Bostjan Golob}

University of Ljubljana and Jožef Stefan Institute, Ljubljana, Slovenia

E-mail: bostjan.golob@fmf.uni-lj.si

\section{Robert Fleischer}

Nikhef and Department of Physics and Astronomy, Vrije Universiteit Amsteram, Amsterdam, Netherlands

E-mail: robert.fleischer@nikhef.nl

\section{Guy Wilkinson}

Department of Physics, University of Oxford, Oxford, United Kingdom

E-mail: guy.wilkinsonecern.ch

\begin{abstract}
The international conference devoted to $b$-hadron physics at frontier machines, Beauty 2019, was held in Ljubljana, Slovenia, from 30 September to 4 October. It was the $18^{\text {th }}$ edition of the series "International Conference on B-Physics at Frontier Machines", initiated in the Czech Republic in 1993. The aims of the conference series are to review the latest results in heavy-flavour physics and discuss future directions. This year's edition attracted around 80 scientists and 65 invited talks, of which 13 were theory based.
\end{abstract}

18th International Conference on B-Physics at Frontier Machines - Beauty2019 -

29 September $/ 4$ October, 2019

Ljubljana, Slovenia 
Ljubljana is well known in the heavy-flavour community for the great impact its physicists have made on the Belle and Belle II experiments, and for accompanying theoretical contributions. It was therefore a highly appropriate location for the Beauty 2019, the second time the conference series had visited Slovenia (the first being to Bled in 1999). It was pleasing to note that almost all registrants attended the meeting, which was remarkable given that only the previous week the national airline had declared bankruptcy, with the consequence that very many flights were cancelled, necessitating new travel arrangements to be made.

The study of hadrons containing beauty quarks, and other heavy flavours, offers a powerful way to probe for physics beyond the Standard Model, as highlighted in the inspiring opening talk by Chris Quigg (Fermilab). In the last few years much attention has been focused on $b$-physics results that do not show perfect agreement with the predictions of the theory. In particular, studies by Belle, BaBar and LHCb of the processes $B \rightarrow K^{-} \ell^{+} \ell^{-}$and $B^{0} \rightarrow K^{*} \ell^{+} \ell^{-}$(where $\ell$ indicates a lepton) in specific kinematic regions have yielded different decay rates for muon pairs and electron pairs, apparently violating lepton universality. For both processes the significance of the effect is around 2.5 sigma. Popular models to explain this and related effects include leptoquarks and new $Z^{\prime}$ bosons, however no firm conclusions can be drawn until more precise measurements are available, which should be the case when the next Beauty meeting occurs.

The $b$-hadron system is an ideal laboratory for the study of $C P$ violation, and recent results were presented by the LHC experiments for $\phi_{s}$, the phase associated with time-dependent measurements of $B_{s}^{0}$-meson decays to $C P$ eigenstates. Indications that $\phi_{s}$ is nonzero are starting to emerge, which is remarkable given that its magnitude in the Standard Model is less than 0.1 radians. This is great encouragement for Run 3 of the LHC, and beyond.

Heavy-flavour experiments are also well suited to the study of hadron spectroscopy. Many very recent results were shown at the conference including the discovery of the $\mathrm{X}(3842)$, which is a charmonium resonance above the open charm threshold, and new excited resonances seen in the $\Lambda_{b} \pi \pi$ final state, which help map out the relatively unexplored world of $b$-baryons. The ATLAS collaboration presented, for the first time, an analysis of $\Lambda_{b} \rightarrow J / \psi p K$ decays in which a structure is observed that is compatible with that of the LHCb pentaquark discovery of 2015, providing the first confirmation by another experiment of these highly exotic states.

The Beauty conference welcomes reports on flavour studies beyond $b$-physics, and a highlight of the week was the first presentation at a conference of new results on the measurement of the branching ratio of the ultra-rare decay $K^{+} \rightarrow \pi^{+} v \bar{v}$, by the NA62 collaboration. The impressive background suppression that the experiment has achieved left the audience in no doubt as to the sensitivity of the result that can be expected when the full data set is accumulated and analysed. Comparing the measurement with the predicted branching fraction of $\sim 10^{-10}$ will be a critical test of the Standard Model in the flavour domain.

The experimental presentations were complemented by theoretical overview talks, summarising recent progress and pointing out open questions. There were interesting discussions on the interplay between theory and experiment in flavour physics, mapping out ways to possible New Physics beyond the Standard Model.

It was pleasing that many excellent presentations on important results were given by young researchers. In the same spirit several interesting posters were displayed by early-career physicists, who each gave an accompanying short presentation in a session dedicated for this purpose. 
Flavour physics has a bright future. Several talks presented the first signals and results from the early running of the Belle II experiment, and precise and exciting measurements can be expected when the next meeting in the Beauty series takes place. In parallel, studies with increasing sensitivity will continue to emerge from the LHC. The meeting was updated about progress on the LHCb Upgrade, which is currently being installed ready for Run 3, and will allow for an order of magnitude increase in b-hadron samples. The conference was summarized by Patrick Koppenburg (Nikhef), who emphasised the enormous potential of $b$-hadron studies for uncovering signs of New Physics beyond the Standard Model.

The formal sessions of the conference were complemented by a diverse social programme, including a reception, a gastronomic walking tour of the city, and a dinner in a brewery. All of these events proved very successful for stimulating both scientific discussion and interactions of a more general nature.

The next edition of Beauty will take place in Japan, hosted by Kavli IPMU, University of Tokyo, autumn 2020.

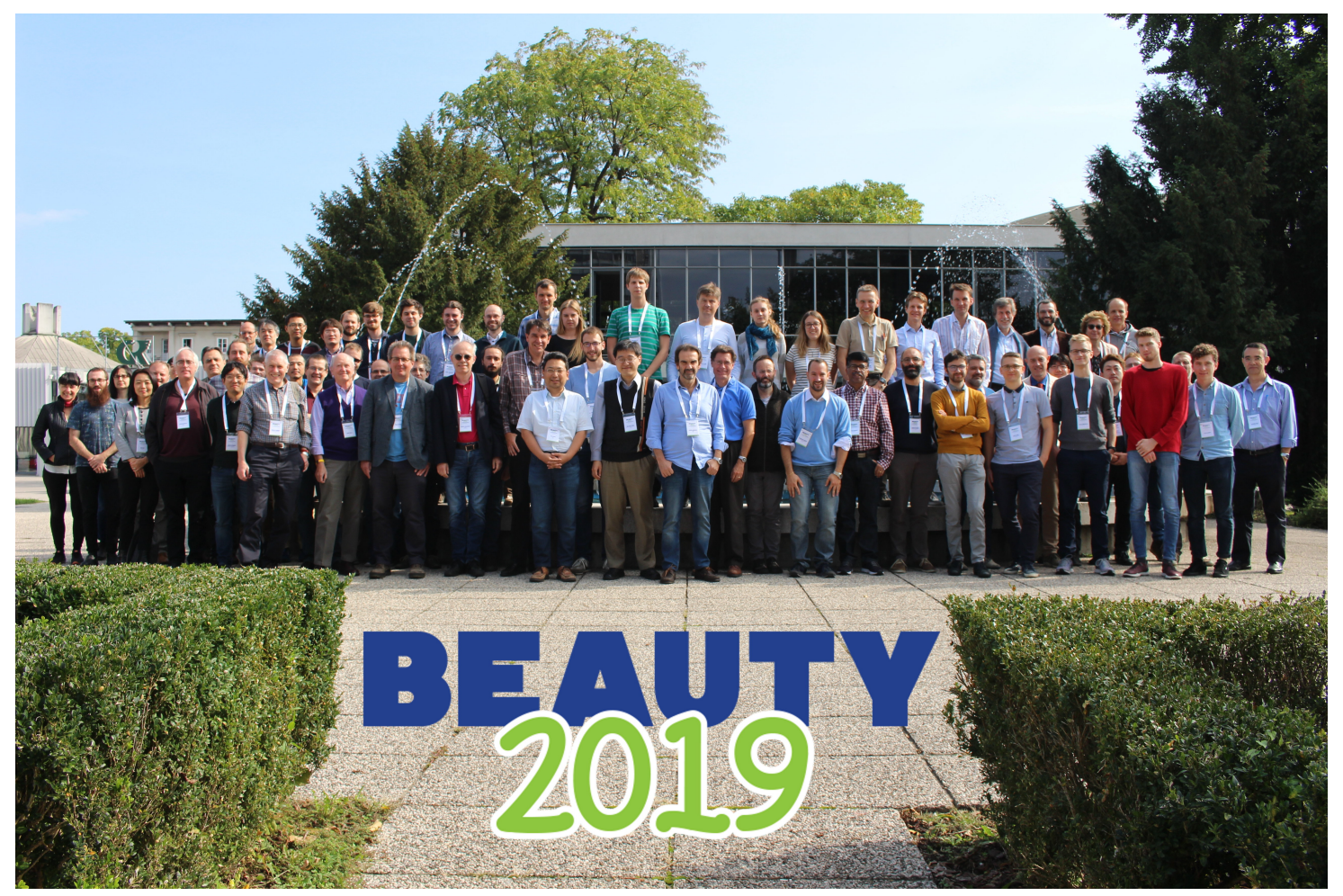

Figure 1: A group photograph of the Beauty 2019 participants outside the conference centre. 